\title{
Constraints on Far-Infrared Source Counts in the Lockman Hole using a Power Spectrum Analysis
}

\author{
H. Matsuhara,
}

Institute of Space and Astronautical Science, Kanagawa, 229 Japan

K. Kawara \& Y. Sofue

Institute of Astronomy, The University of Tokyo, Tokyo, 181-8588, Japan

Y. Taniguchi

Astronomical Institute, Tohoku University, Sendai 980-8578, Japan

Y. Sato, H. Okuda, \& T. Matsumoto

The Institute of Space and Astronautical Science, Kanagawa, 229-8510, Japan

K. Wakamatsu

Faculty of Engineering, Gifu University, Gifu 501-1193, Japan

L. L. Cowie, R. D. Joseph, \& D. B. Sanders

Institute for Astronomy, University of Hawaii, Honolulu, HI 96822, USA

Abstract. We present the characteristics of far-infrared(FIR) brightness fluctuations at $90 \mu \mathrm{m}$ and $170 \mu \mathrm{m}$ in the Lockman Hole, which were surveyed with the ISOPHOT instrument aboard the Infrared Space Observatory (ISO), and give constraints on the galaxy number counts down to $30 \mathrm{mJy}$ at $90 \mu \mathrm{m}$ and $50 \mathrm{mJy}$ at $170 \mu \mathrm{m}$. The fluctuation power spectra of the FIR images are not dominated by IR cirrus, and are instead most likely due to star-forming galaxies. This analysis indicates the existence of strong evolution in the counts. Especially at $90 \mu \mathrm{m}$, the source density is much larger than that expected from the currently available galaxy count models. The galaxies responsible for the fluctuations also significantly contribute to the cosmic infrared background radiation recently derived from an analysis of the COBE data. 